\title{
Galaxy Clusters: Cosmic High-Energy Laboratories to Study the Structure of Our Universe
}

\author{
Hans Böhringer \\ Max-Planck-Institut für Extraterrestrische Physik, D-85748 Garching, Germany
}

\begin{abstract}
This contribution illustrates the study of galaxy clusters as astrophysical laboratories as well as probes for the large-scale structure of the Universe. Using the REFLEX Cluster Survey, the measurement of the statistics of the large-scale structure on scales up to $500 h^{-1} \mathrm{Mpc}$ is illustrated. The results clearly favour a low density Universe.

Clusters constitute, in addition, well defined astrophysical laboratory environments in which some very interesting large-scale phenomena can be studied. As an illustration we show some spectacular new XMM X-ray spectroscopic results on the thermal structure of cooling flows and the interaction effects of AGN with this hot intracluster medium. The X-ray observations with XMM-Newton show a lack of spectral evidence for large amounts of cooling and condensing gas in the centers of galaxy clusters believed to harbour strong cooling flows. To explain these findings we consider the heating of the core regions of clusters by jets from a central AGN. We find that the power output the AGN jets is well sufficient. The requirements such a heating model has to fulfill are explored and we find a very promising scenario of self-regulated Bondi accretion of the central black hole.
\end{abstract}

\section{Introduction}

Galaxy clusters with masses from about $10^{14}$ to over $10^{15} \mathrm{M}_{\odot}$ are the largest clearly defined building blocks of our universe. Their formation and evolution is tightly connected to the evolution of the large-scale structure of our Universe as a whole. Clusters are therefore ideal probes for the study of the large-scale matter distribution. Due to the hot, intracluster plasma trapped in the potential well of galaxy clusters, which can take on temperatures of several keV (several 10 Million degrees), galaxy clusters are the most luminous X-ray emitters in the Universe next only to quasars. The hot gas and its X-ray emission is a good tracer of the gravitational potential of the clusters and thus allows us to obtain mass estimates of the clusters, to study their morphology and dynamical state, and to detect clusters as gravitationally bound entities out to very large distances (Sarazin 1986). Systematic studies have shown that clusters have within a first order description, a quite standardized, self-similar appearance. This is reflected observationally in quite narrow correlations of observable parameters, like e.g. the temperature - X-ray luminosity relation. The for our application most important relation is that of mass and X-ray luminosity. Since, for the construction of a cosmologically interesting cluster sample, we are interested to 
collect all the clusters above a certain mass limit, this relation makes it possible to select the most massive clusters essentially by their X-ray luminosity.

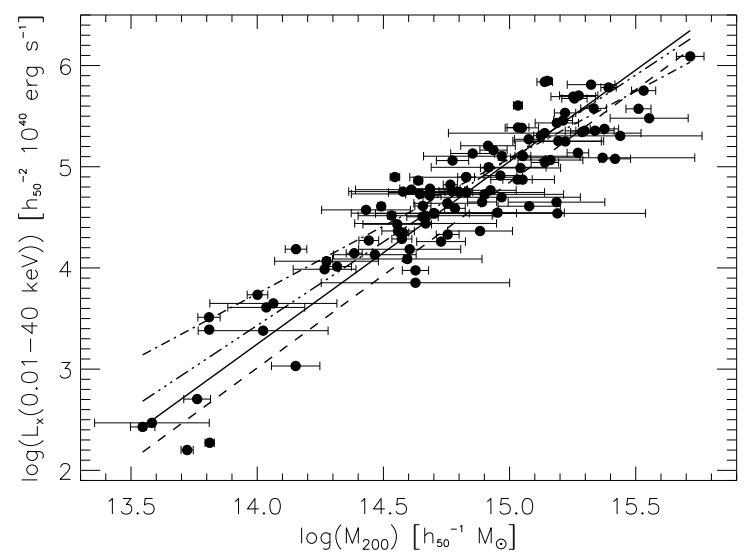

Fig. 1. Mass-X-ray luminosity relation determined from the 106 brightest galaxy clusters found in the ROSAT All-Sky Survey by Reiprich \& Böhringer (1999, 2001)

The first detailed mass-X-ray luminosity relation compiled from X-ray observations is shown in Fig.1 (Reiprich \& Böhringer 1999, 2001). From these results a cluster mass function and its integral, the matter density in the Universe bound in clusters was derived. In Fig. 2 we show this cumulative cluster mass density function, yielding $\Omega_{\text {clusters }} \sim 0.02$. Thus for the currently most popular value for the matter density $\Omega_{m} \sim 0.3$ about $6 \%$ of the matter in the Universe is bound in clusters with a mass larger than $6.4 \cdot 10^{13} h_{50}^{-1} \mathrm{M}_{\odot}$.

\section{Measurement of the Large-Scale Structure}

Based on the ROSAT All-Sky X-ray Survey - so far the only all-sky survey conducted with an X-ray telescope - we exploit the above mentioned tight X-ray luminosity-mass relation with X-ray selected cluster redshift surveys to study the large-scale structure of the Universe. Fig.3 shows the sky distribution of the brightest X-ray galaxy clusters identified so far in the ROSAT All-Sky Survey within two projects: the northern NORAS Survey (Böhringer et al. 2000) and the southern REFLEX Survey (Böhringer et al. 2001a). The latter project is currently more complete comprising a sample of 452 clusters and therefore the following results are based on this project. Most of the redshifts for this survey have been obtained within the frame of an ESO key program (Böhringer et al. 1998, Guzzo et al. 1999) .

The basic census of the REFLEX cluster survey is the X-ray luminosity function shown in Fig. 4 (Böhringer et al. 2002). The essential goal of this survey is 


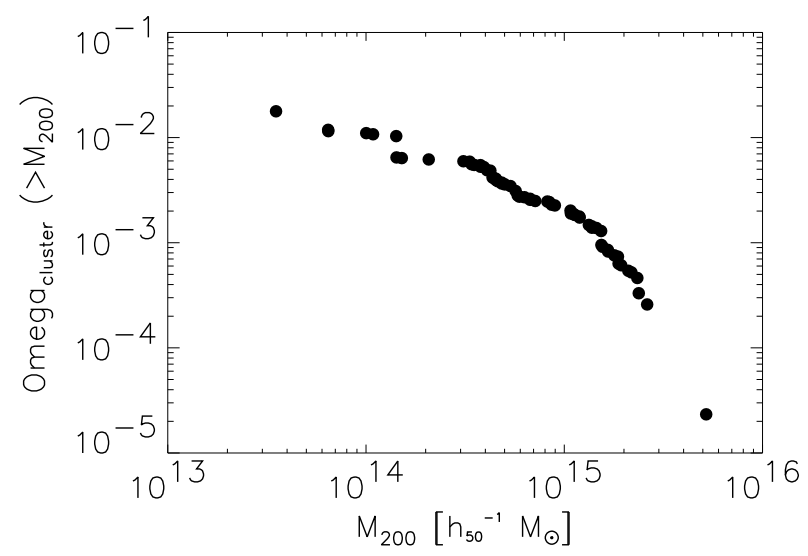

Fig. 2. Cumulative fraction of the mass density bound in clusters in the Universe compared to the critical density (Reiprich \& Böhringer 2001)

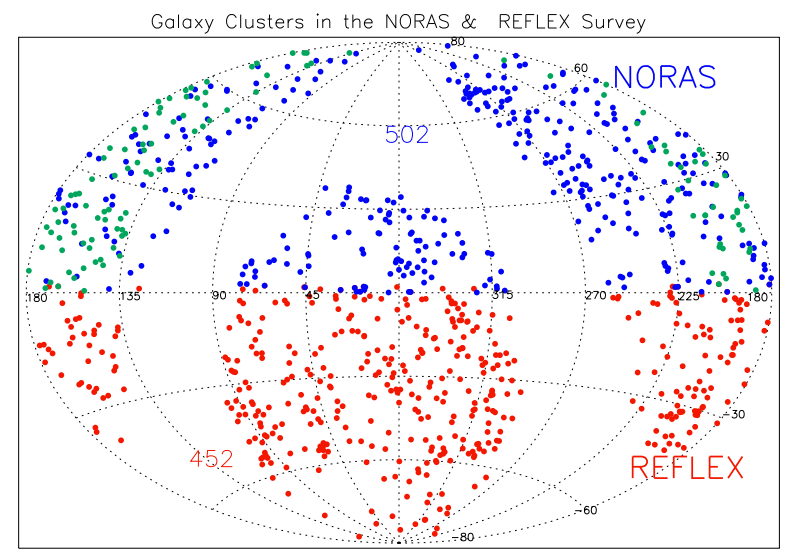

Fig. 3. Sky distribution of the brightest galaxy clusters found in the ROSAT All-Sky Survey investigated in the NORAS and REFLEX redshift surveys.

the assesment of the statistics of the large-scale structure. The most fundamental statistical description of the spatial structure is based on the second moments on the distribution, characterized either by the two-point-correlation function or its Fourier transform, the density fluctuation power spectrum. The two-point correlation function of the clusters in the REFLEX sample has been derived by Collins et al. (2000). The results show a power law shaped correlation function with a slope of 1.83 , a correlation length of $18.8 h_{100}^{-1} \mathrm{Mpc}$ and a possible zero crossing at $\sim 45 h_{100}^{-1} \mathrm{Mpc}$. The density fluctuation power spectrum (Fig. 5) has been determined by Schuecker et al. (2001a). The power spectrum is character- 
ized by a power law at large values of the wave vector, $k$, with a slope of $\propto k^{-2}$ for $k \leq 0.1 h \mathrm{Mpc}^{-1}$ and a maximum around $k \sim 0.03 h \mathrm{Mpc}^{-1}$ (corresponding to a wavelength of about $200 h^{-1} \mathrm{Mpc}$ ). This maximum reflects the size of the horizon when the Universe featured equal energy density in radiation and matter and is a sensitive measure of the mean density of the Universe, $\Omega_{0}$. For standard open and flat Cold Dark Matter models (OCDM and $\Lambda \mathrm{CDM}$ ) we find the following constraints $h \Omega_{0}=0.12$ to 0.26 (Schuecker et al. 2001a).

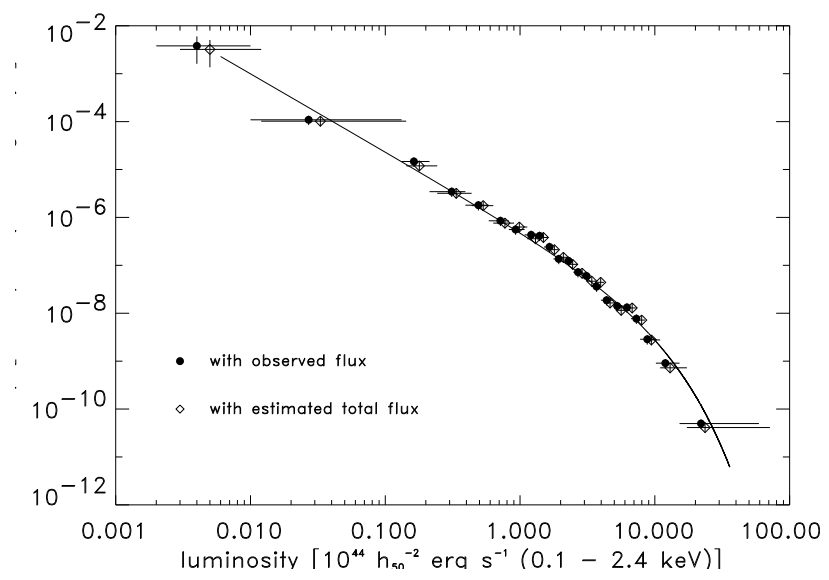

Fig. 4. X-ray luminosity function of the REFLEX cluster Survey (Böhringer et al. 2002). The two series of data point refer to the calculation of the luminosities from the X-ray fluxes as observed and corrected for the missing flux in the outer very low surface brightness regions, respectively.

\section{Cosmological Tests with Galaxy Clusters}

In addition to the above mentioned large-scale structure test for cosmological models several further studies yield information on cosmological parameters. These include the modelling of the mass, temperature, and luminosity functions (e.g. Reiprich \& Böhringer 2001, Ikebe et al. 2001), the study of the mass-tolight ratio in connection with galaxy biasing Carlberg et al. (1996; which yields $\Omega_{0} \sim 0.24 \pm 0.1$ ), the baryon mass fraction in clusters, and the statistics of merging clusters as observed in the REFLEX cluster sample (Schuecker et al. 2001b). All these different cosmological studies using clusters point towards a low density universe. These results can be compared to the results obtained from observations of the cosmic microwave anisotropies (e.g. De Bernardis et al. 2000, 2001) and of the study of distant SN Ia (e.g. Perlmutter et al. 1999). While these two investigations provide combined constraints that encircle a region in the model parameter space spanned by the cosmological parameters $\Omega_{0}$ and $\Omega_{\Lambda}$ 


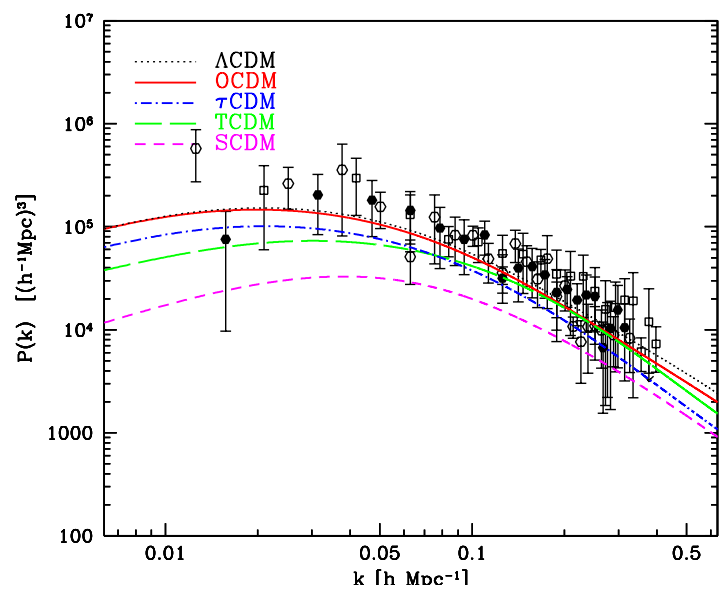

Fig. 5. Power spectra of the density fluctuations in the REFLEX cluster sample together with predictions from various popular cosmological models taken from the literature. For details see Schuecker et al. (2001a).

around values of $\Omega_{0}=0.3$ and $\Omega_{\Lambda}=0.7$, the galaxy cluster results provide a different cut through this parameter space crossing the other two results at their intersection. That is, the cluster results provide at present (without the inclusion of an investigation of a very large redshift range) no significant constraints on the $\Omega_{\Lambda}$-parameter, but allow values of for $\Omega_{0}=0.2-0.4$ in the range consistent with the combined cosmic microwave and SN Ia data. Thus, the evidence for a low density universe is solidifying.

\section{Cluster Cooling Cores}

X-ray imaging observations have shown that the X-ray emitting, hot gas in a large fraction of all galaxy clusters reaches high enough densities in the cluster centers that the cooling time of the gas falls below the Hubble time, and gas may cool and condense in the absence of a suitable fine-tuned heating source (e.g. Silk 1976, Fabian \& Nulsen 1977). From the detailed analysis of surface brightness profiles of X-ray images of clusters obtained with the Einstein, EXOSAT, and ROSAT observatories, the detailed, self-consistent scenario of inhomogeneous, comoving cooling flows emerged (e.g. Nulsen 1986, Thomas, Fabian, \& Nulsen 1987, Fabian 1994). The main assumptions on which the cooling flow model is based and some important implications are: (i) Each radial zone in the cooling 
Hans Böhringer et al.

flow region comprises different plasma phases covering a wide range of temperatures. The consequence of this temperature distribution is that gas will cool to low temperature and condense over a wide range of radii. (ii) The gas features an inflow in which all phases with different temperature move with the same flow speed. (iii) There is no energy exchange between the different phases, between material at different radii, and no heating.

Now the first analysis of high resolution X-ray spectra and imaging spectroscopy obtained with XMM-Newton has shown to our surprise that the spectra show no signatures of cooler phases of the cooling flow gas below an intermediate temperature (e.g. Peterson et al. 2001, Tamura et al. 2001) and local isothermality in the cooling flow region (e.g. Böhringer et al. 2001b, Matsushita et al. 2001, Molendi \& Pizzolato 2001) in conflict with the inhomogeneous cooling flow model. Here, we discuss these new spectroscopic results and their implications and point out the way to a new possible model for this phenomenon. The results are mostly based on the detailed observations of the M87 X-ray halo. A detailed description of this study is provided by Böhringer et al. (2001c).

\section{Spectroscopic Diagnostics of Cluster Cooling Cores}

XMM Reflection Grating Spectrometer (RGS) observations of several cooling core regions show signatures of different temperature phases ranging approximately from the hot virial temperature of the cluster to a lower limiting temperature, $T_{\text {low }}$. Clearly observable spectroscopic features of even lower temperature gas expected for a cooling flow model are not observed. A1835 with a bulk temperature of about $8.3 \mathrm{keV}$ has $T_{\text {low }}$ around $2.7 \mathrm{keV}$ (Peterson et al. 2001) and similar results have been derived for A1795 (Tamura et al. 2001). These results are very well confirmed by XMM observations with the energy sensitive imaging devices, EPN and EMOS, providing spectral information across the entire cooling core region, yielding the result that (for M87, A1795, and A1835) single temperature models provide a better representation of the data than cooling flow models (Böhringer et al. 2001a, Molendi \& Pizzolato 2001) also implying the lack of low temperature components. The very detailed analysis of M87 by Matsushita et al. (2001 and the contribution to this workshop) has shown that the temperature structure is well described locally by a single temperature over most of the cooling core region, except for the regions of the radio lobes and the very center $(r \leq 1 \mathrm{arcmin}, \sim 5 \mathrm{kpc})$.

Among the spectroscopic signatures which are sensitive to the plasma temperature in the relevant temperature range, the complex of iron L-shell lines is most important. Fig. 6 shows simulated X-ray spectra as predicted for the XMM EPN instrument in the spectral region around the Fe L-shell lines for a single-temperature plasma at various temperatures from 0.4 to $2.0 \mathrm{keV}$ and 0.7 solar metallicity. There is a very obvious shift in the location of the peak making this feature an excellent thermometer. For a cooling flow with a broad range of temperatures one expects a composite of several of the relatively narrow line blend features, resulting in a quite broad peak. Fig. 7a shows for example the 


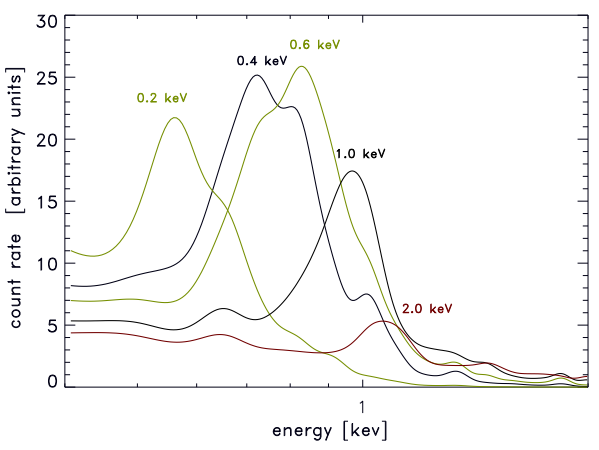

Fig. 6. The Fe L-line complex in X-ray spectra as a function of the plasma temperature for a metallicity value of 0.7 solar. The simulations show the appearance of the spectra as seen with the XMM EPN. The emission measure was kept fixed when the temperature was varied.

deprojected spectrum of the M87 halo plasma for the radial range 1 - 2 arcmin (outside the inner radio lobes) and a fit of a cooling flow model with a mass deposition rate slightly less than $1 \mathrm{M}_{\odot} \mathrm{yr}^{-1}$ as expected for this radial range from the analysis of the surface brightness profile (e.g. Matsushita et al. 2001). It is evident that the peak in the cooling flow model is much broader than the observed spectral feature. For comparison Fig. 7b shows the same spectrum fitted by a cooling flow model where a temperature of $2 \mathrm{keV}$ was chosen for the maximum temperature and a suitable lower temperature cut-off $(1.44 \mathrm{keV})$ was determined by the fit. The very narrow temperature interval (almost isothermality) is well consistent with the narrow peak. A similar result is obtained for other clusters, e.g. A1795 as shown in Fig. 7d.

Since this diagnostics of the temperature structure is essentially based on the observation of metal lines, an inhomogeneous distribution of the metal abundances in the cluster ICM and a resulting suppression of line emission at low temperatures was suggested as a possible way to reconcile the above findings with the standard cooling flow model by Fabian et al. (2001 and contribution in these proceedings). As shown by Böhringer et al. (2001c) such a scenario will still result in a relatively broad Fe L-line feature and does not solve the problem in this case of M87.

\section{Internal absorption}

Another possible attempt to obtain consistency is to allow the absorption parameter in the fit to adjust freely. This is demonstrated in Fig. 7c with the same observed spectrum where the best fitting absorption column density is selected in such a way by the fit that the absorption edge limits the extent of the $\mathrm{Fe}-$ $\mathrm{L}$ line feature towards lower energies. This is actually the general finding with ASCA observations showing two possible options for the interpretation of the 
spectra of cluster core regions: (1) an interpretation of the results in form of an inhomogeneous cooling flow model which than necessarily includes an internal absorption component (e.g. Allen 2000, Allen et al. 2001), or (2) an explanation of the spectra in terms of a two-temperature component model (e.g. Ikebe et al. 1999, Makishima 2001) where the hot component is roughly equivalent to the hot bulk temperature of the clusters and the cool component corresponds approximately to $T_{\text {low }}$. Thus for the cooling flow interpretation to work and to produce a sharp Fe-L line feature as observed, the absorption edge has to appear at the right energy and therefore values for the absorption column of typically around $3 \cdot 10^{21} \mathrm{~cm}^{-2}$ are needed (e.g. Allen 2000 and Allen et al. 2001 who find values in the range $\left.1.5-5 \cdot 10^{21} \mathrm{~cm}^{-2}\right)$.

It is therefore important to perform an independent test on the presence of absorbing material in the cluster cores. Thanks to CHANDRA and XMMNewton we can now use central cluster AGN as independent light sources for probing. Using the nucleus and jet of M87 (with XMM, see Fig. 3) and the nucleus of NGC1275 (with CHANDRA) we find no signature of internal absorption. Thus at least for these two cases internal absorption is not observed.
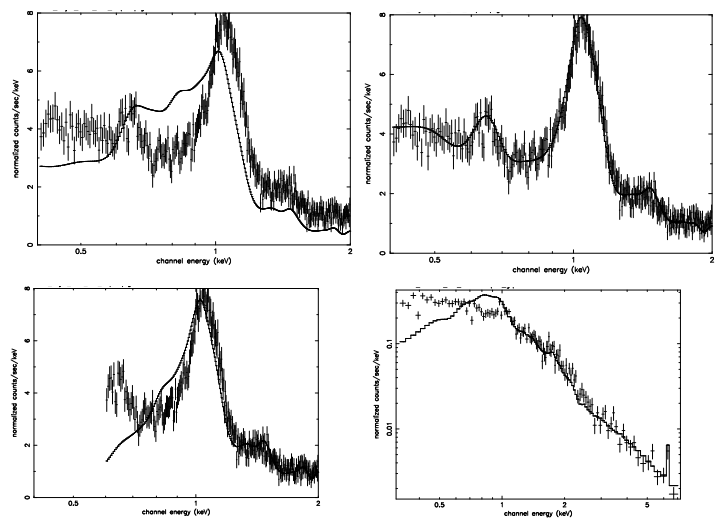

Fig. 7. XMM EPN spectrum of the M87 X-ray halo in the radial range $R=1-2$ arcmin. The spectrum has been fitted with a cooling flow model with a best fitting mass deposition rate of $0.96 \mathrm{M}_{\odot} \mathrm{yr}^{-1}$ and a fixed absorption column density of $1.8 \cdot 10^{20}$ $\mathrm{cm}^{-2}$, the galactic value, and a parameter for $T_{l o w}$ of $0.01 \mathrm{keV}$. (b - upper right): same spectrum fitted by a cooling flow spectrum artificially constraint to emission from the narrow temperature interval $1.44-2.0 \mathrm{keV}$, where $T_{\text {low }}$ was treated as a free fitting parameter. (c - lower left): same spectrum fitted with a free parameter for the internal excess absorption. The spectrum was constraint to the energy interval 0.6 to $2.0 \mathrm{keV}$. (d - lower right): XMM EPN spectrum of A1795 fitted with a cooling flow model with the galactic value for absorption. 


\section{Heating Model}

In view of these difficulties of interpreting the observations with the standard cooling flow model, we may consider the possibility that the cooling and mass deposition rates are much smaller than previously thought, that is reduced by at least one order of magnitude. To decrease the mass condensation under energy conservation some form of heating is clearly necessary. Three forms of heat input into the cooling flow region have been discussed: (i) heating by the energy output of the central AGN (e.g. Pedlar et al. 1990, Tabor \& Binney 1993, McNamara et al. 2000, (ii) heating by heat conduction from the hotter gas outside the cooling flow (e.g. Tucker \& Rosner 1983, Bertschinger \& Meiksin 1986), and (iii) heating by magnetic fields, basically through some form of reconnection (e.g. Soker \& Sarazin 1990, Makishima et al. 2001). The latter two processes depend on poorly known plasma physical conditions and are thus more speculative. The energy output of the central AGN, however, can be determined as shown below.

A heating scenario can only successfully explain the observations if among others the two most important requirements are met: (i) The energy input has to provide sufficient heating to balance the cooling flow losses, that is about $10^{60}$ to $10^{61} \mathrm{erg}$ in $10 \mathrm{Gyr}$ or on average about $3 \cdot 10^{43}-3 \cdot 10^{44} \mathrm{erg} \mathrm{s}^{-1}$, and (ii) The energy input has to be fine-tuned. Too much heating would result in an outflow from the central region and the central regions would be less dense than observed. Too little heat will not reduce the cooling flow by a large factor. Therefore the heating process has to be self-regulated: mass deposition triggers the heating process and the heating process reduces the mass deposition.

Further constraints are discussed by Böhringer et al. (2001c). The total energy input into the ICM by the relativistic jets of the central AGN can be estimated by the interaction effect of the jets with the ICM by means of the scenario described in Churazov et al. (2000). It relies on a comparison of the inflation and buoyant rise time of the bubbles of relativistic plasma which are observed e.g. in the case of NGC 1275 (Böhringer et al. 1993, Fabian et al. 2000).

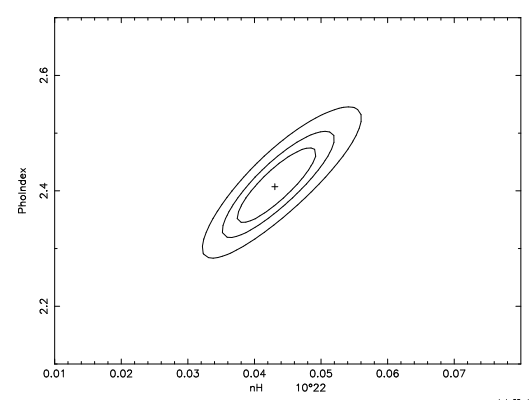

Fig. 8. Constraints on the shape of the XMM EPN-spectrum of the nucleus of M87. The lines show the 1,2 , and $3 \sigma$ confidence intervals for the combined fit of the slope (photon index) of the power law spectrum and the value for the absorbing column density, $n_{H}$, in units of $10^{22} \mathrm{~cm}^{-2}$. 
The estimated total energy output is for three examples, M87: $1.2 \cdot 10^{44} \mathrm{erg} \mathrm{s}^{-1}$, Perseus: $1 \cdot 10^{45} \mathrm{erg} \mathrm{s}^{-1}$, and Hydra A: $2 \cdot 10^{45} \mathrm{erg} \mathrm{s}^{-1}$. These values for the energy input have to be compared with the energy loss in the cooling flow, which is of the order of $10^{43} \mathrm{erg} \mathrm{s}^{-1}$ for M87 and about $10^{44} \mathrm{erg} \mathrm{s}^{-1}$ for Perseus. Thus in these cases the energy input is larger than the radiation losses in the cooling flow for at least about the last $10^{8}$ yr. We have, however, evidence that this energy input continued for a longer time with evidence given by the outer radio halo around M87 with an outer radius of $35-40 \mathrm{kpc}$ (e.g. Kassim et al. 1993, Rottmann et al. 1996). Owen et al. (2000) give a detailed physical account of the halo and model the energy input into it. They estimate the total current energy content in the halo in form of relativistic plasma to $3 \cdot 10^{59} \mathrm{erg}$ and the power input for a lifetime of about $10^{8}$ years, which is also close to the lifetime of the synchrotron emitting electrons, to the order of $10^{44} \mathrm{erg} \mathrm{s}^{-1}$, consistent with our estimate. The very characteristic sharp outer boundary of the outer radio halo of M87, noted by Owen et al. (2000), has the important implications, that this could not have been produced by magnetic field advection in a cooling flow.

Thus, we find a radio structure providing evidence for a power input from the central AGN into the halo region of the order of about ten times the radiative energy loss rate over at least about $10^{8}$ years (for M87). The energy input could therefore balance the heating for at least about $10^{9}$ years. The observation of active AGN in the centers of cooling flows is a very common phenomenon. E.g. Ball et al. (1993) find in a systematic VLA study of the radio properties of cD galaxies in cluster centers, that $71 \%$ of the cooling flow clusters have radio loud cDs compared to $23 \%$ of the non-cooling flow cluster cDs. Therefore we can safely assume that the current episode of activity was not the only one in the life of M87 and its cooling flow.

The mechanism for a fine-tuned heating of the cooling flow region should most probably be searched for in a feeding mechanism of the AGN by the cooling flow gas. The most simple physical situation would be given if simple Bondi type of accretion from the inner cooling core region would roughly provide the order of magnitude of the power output that is observed and required. Using the classical formula for spherical accretion from a hot gas by Bondi (1952) we can obtain a very rough estimate for this number. For the proton density near the M87 nucleus $(r \leq 15 \operatorname{arcsec})$ of about $0.1 \mathrm{~cm}^{-3}$, a temperature of about $10^{7} \mathrm{~K}$ (e.g. Matsushita et al. 2001), and a black hole mass of $3 \cdot 10^{9} \mathrm{M}_{\odot}$ (e.g. Ford et al. 1994) we find a mass accretion rate of about $0.01 \mathrm{M}_{\odot} \mathrm{yr}^{-1}$ and an energy output of about $7 \cdot 10^{43} \mathrm{erg} \mathrm{s}^{-1}$, where we have assumed the canonical value of 0.1 for the ratio of the rest mass accretion rate to the energy output. The corresponding accretion radius is about $50 \mathrm{pc}(\sim 0.6 \operatorname{arcsec})$. This accretion rate is more than a factor of 1000 below the Eddington value and thus no reduction effects of the spherical accretion rate by radiation pressure has to be expected. Small changes in the temperature and density structure in the inner cooling core region will directly have an effect on the accretion rate. Therefore we have all the best prospects for building a successful self-regulated AGN-feeding and cooling flow-heating model. 


\section{Conclusions}

Several observational constraints have let us to the conclusion that the mass deposition rates in galaxy cluster cooling cores are not as high as previously predicted. The new X-ray spectroscopic observations with a lack of spectral signatures for the coolest gas phases expected for cooling flows and the lower mass deposition rates indicated at other wavelength bands than X-rays are more consistent with mass deposition rates reduced by one or two orders of magnitude below the previously derived values. This can, however, only be achieved if the gas in the cooling flow region is heated. The most promising heating model is a self-regulated heating model powered by the large energy output of the central AGN in most cooling flows. for cooling flows and the lower mass deposition rates indicated at Most of the guidance and the support of the heating model proposed here (based on concepts developed in Churazov et al. 2000, 2001) is taken from the detailed observations of a cooling core region in the halo of M87 and to a smaller part from the observations in the Perseus cluster. These observations show that the central AGN produces sufficient heat for the energy balance of the cooling flow, that the most fundamental and classical accretion process originally proposed by Bondi (1952) provides an elegant way of devising a self-regulated model of AGN heating of the cooling flow, and that most of the further requirements that have to be met by a heating model to be consistent with the observations can most probably be fulfilled. Since these ideas are mostly developed to match the conditions in M87, it is important to extent such detailed studies to most other nearby cooling flow clusters.

In this new perspective the cooling cores of galaxy clusters become the sites where most of the energy output of the central cluster AGN is finally dissipated. Strong cooling flows should therefore be the locations of AGN with the largest mass accretion rates. While in the case of M87 with a possible current mass accretion rate of about $0.01 \mathrm{M}_{\odot} \mathrm{y}^{-1}$ the mass addition to the black hole (with an estimated mass of about $3 \cdot 10^{9} \mathrm{M}_{\odot}$ ) is a smaller fraction of the total mass, the mass build-up may become very important for the formation of massive black holes in the most massive cooling flows, where mass accretion rates above 0.1 $\mathrm{M}_{\odot} \mathrm{y}^{-1}$ become important over cosmological times.

I like to thank the ROSAT team, the ESO key program team, the NORAS team, and in particular Peter Schuecker, Luigi Guzzo, Chris Collins, Kyoko Matsushita, Eugene Churazov, Yasushi Ikebe, Thomas Reiprich, and Yasuo Tanaka

for the pleasant and fruitful collaboration. I like to thank in particular Rashid Sunyaev for the organization of this very magnificent conference!

\section{References}

1. Allen, S.W., 2000, MNRAS, 315, 269

2. Allen, S.W., Fabian, A.C., Johnstone, R.M., et al., 2001, MNRAS, 322, 589

3. Ball, R., Burns, J.O., Loken, C., 1993, AJ, 105, 53

4. Bertschinger, E. \& Meiksin, A., 1986, ApJ, 306, L1 
5. Böhringer, H., Voges, W., Fabian, A.C. Edge, A.C., and Neumann, D., 1993, MNRAS, 264, L25

6. Böhringer, H., Guzzo, L., Collins, C.A., et al. 1998, The Messenger, No. 94, 21

7. Böhringer, H., Voges, W., Huchra, J.P., et al., 2000, ApJS, 129, 435

8. Böhringer, H., Schuecker, P., Guzzo, L., et al., 2001a, A\&A, 369, 826

9. Böhringer, H., Belsole, E., Kennea, J., et al., 2001b, A\&A, 365, L181

10. Böhringer, H., Matsushita, K., Ikebe, Y., et al. 2001c, A\&A, accepted

11. Böhringer, H., Collins, C.A., Guzzo, L., 2002, ApJ, 566, 1

12. Bondi, H., 1952, MNRAS, 112, 195

13. Carlberg, R.G., Yee, H.K.C., Ellingson, E., et al. 1996, ApJ, 462, 32

14. Collins, C.A., Guzzo, L., Böhringer, H., 2000, MNRAS, 319, 939

15. Churazov, E., Forman, W., Jones, C., Böhringer, H., 2000, A\&A, 356, 788

16. Churazov, E., Brüggen, M., Kaiser, C.R., Böhringer, H., \& Forman, W., 2001, ApJ, $\mathbf{5 5 4}, 261$

17. De Bernardis et al., 2000, Nature, 404, 955

18. De Bernardis et al., 2001, astro-ph/0105296

19. Fabian, A.C., 1994, ARA\&A, 32, 277

20. Fabian, A. C., Sanders, J. S., Ettori, S., et al. 2000, MNRAS 318, 65

21. Fabian, A.C., Mushotzky, R.F., Nulsen, P.E.J., Peterson, J.R., 2001, MNRAS, 321, 20

22. Ford, H.C., et al. 1994, ApJ, 435, L27

23. Guzzo, L., Böhringer, H., Schuecker, P., et al., 1999, The Messenger, No. 95, 27

24. Ikebe, Y., Makishima, K., Fukazawa, Y., et al., 1999, ApJ, 525, 58

25. Ikebe, Y., Reiprich, T.H., Böhringer, H., Tanaka, Y., 2001, A\&A submitted

26. Kassim, N., Perley, R.A., Erickson, W.C., Dwarakanath, K.S., 1993, AJ, 106, 2218

27. Makishima, K., Ezawa, H., Fukazawa, Y., et al., 2001, PASJ, 53, 401

28. Matsushita, K., Belsole, E., Finoguenov, A., Böhringer, H., 2001, A\&A, submitted

29. McNamara, B., Wise, M., Nulsen, P.E.J., et al. 2000, ApJ, 534, L135

30. Molendi, S. \& Pizzolato, F., 2001, ApJ (in press), astro-ph/0106552

31. Nulsen, P.E.J., 1986, MNRAS, 221, 377

32. Owen, F.N., Eilek, J.A., Kassim, N.E., 2000, ApJ, 543, 611

33. Pedlar, A., Ghataure, H. S., Davies, R. D., et al., 1990, MNRAS, 246, 477

34. Perlmutter, N., 1999, ApJ, 517, 565

35. Peterson, J.R., Paerels, F.B.S., Kaastra, J.S., et al., 2001, A\&A, 365, L104

36. Reiprich \& Böhringer, 1999, Astron. Nachr., 320, 296

37. Reiprich \& Böhringer, 2001, ApJ, submitted

38. Rottmann, H., Mack, K.-H., Klein, U., Wielebinski, R., 1996, A\&A, 309, L9

39. Sarazin, C.L., 1986, Rev. Mod. Phys., 58, 1

40. Schuecker, P., Böhringer, H., Guzzo, et al., 2001a, A\&A, 368, 86

41. Schuecker, P., Böhringer, H., Reiprich, T.H., Ferreti, L., 2001b, A\&A in press

42. Silk, J., 1976, ApJ., 208, 646

43. Soker, N. \& Sarazin, C.L., 1990, ApJ, 348, 73

44. Tabor, G. \& Binney, J., 1993, MNRAS, 263, 323

45. Tamura, T., Kaastra, J.S., Peterson, J.R., et al., 2001, A\&A, 365, L87

46. Thomas, P.A., Fabian, A.C., \& Nulsen, P.E.J., 1987, MNRAS, 228, 973

47. Tucker, W.H. \& Rosner, R., 1983, ApJ, 267, 547 